\title{
Präventive Irritationen in der Dogmatik des Strafprozessrechts
}

\section{Peter Albrecht *}

Das schweizerische Strafprozessrecht ist heute durch ein Sicherheitskonzept geprägt, das die Grenzen zwischen Repression und Prävention verwischt. Besonders deutlich erkennbar wird das beim forcierten Ausbau der präventiven prozessualen Zwangsmassnahmen, so namentlich in den Bereichen der Untersuchungshaft sowie der verdeckten Ermittlung und Fahndung. Die ziemlich radikale Neuorientierung im Prozessrecht (wie auch im Polizeirecht) widerspricht teilweise der von der Verfassung vorgegebenen Kompetenzordnung. Vor allem aber verbindet sich damit ein drastischer Freiheitsverlust für die von den staatlichen Interventionen betroffenen Personen, indem die eingriffsfreien Zonen immer enger werden.

I. Kriminalpolitische Erschütterungen ........................................................59

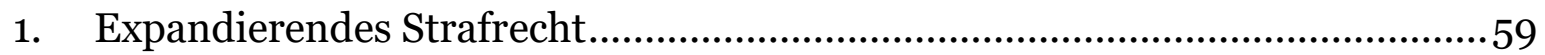

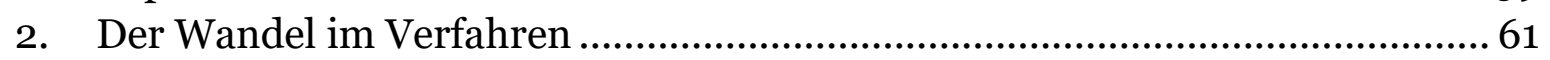

II. Funktionen des Prozessrechts ...................................................................6 63

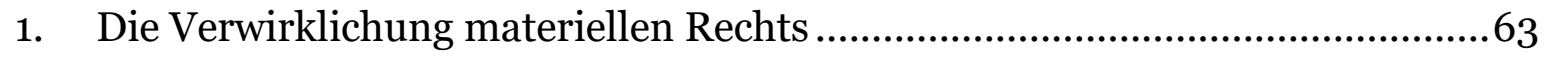

2. Eigenständige Verfahrensinteressen .......................................................64

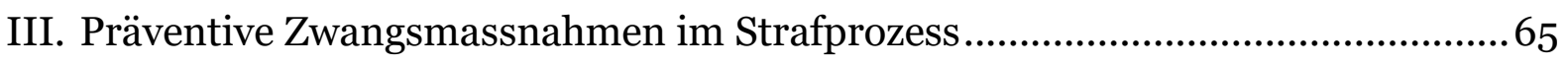

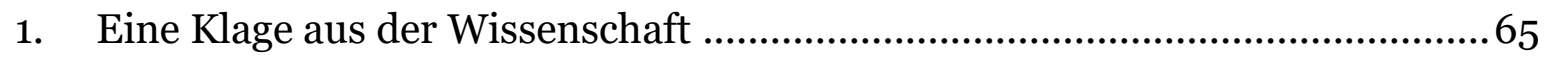

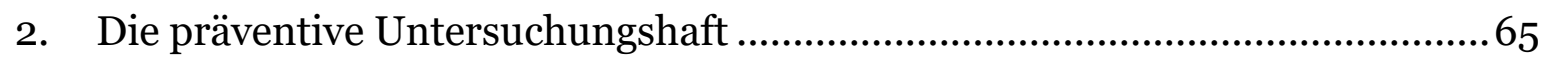

3. Die präventive verdeckte Polizeitätigkeit .................................................. 72

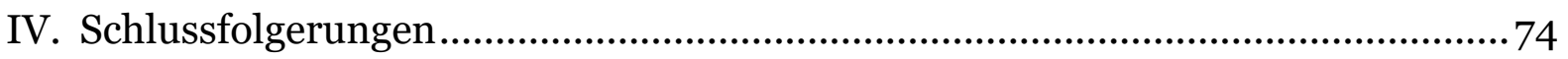

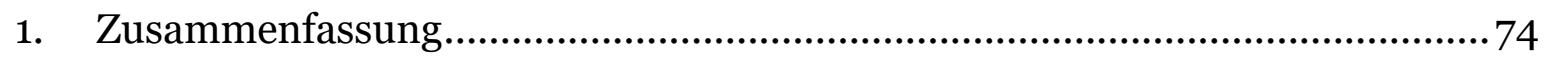

2. Mut zur Lücke! .............................................................................. 75

Zitiervorschlag: Peter Albrecht, Präventive Irritationen in der Dogmatik des Strafprozessrechts, in: sui-generis 2018, S. 58

URL: $\quad$ sui-generis.ch/59

DOI: $\quad$ https://doi.org/10.21257/sg.59

* Prof. Dr. Peter Albrecht, emeritierter Extraordinarius für Strafrecht und Strafverfahrensrecht an der Universität Basel und ehemaliger Strafgerichtspräsident in Basel. 


\section{Kriminalpolitische Erschütterungen}

\section{Expandierendes Strafrecht}

\section{a) Symbolische Steuerung}

1

Die gegenwärtigen rechtspolitischen Entwicklungen in der Schweiz belegen überaus deutlich - und zwar schon seit einigen Jahren -, dass das Strafrecht als verheissungsvolles soziales Instrumentarium gilt und dementsprechend Hochkonjunktur hat. Die Legislative agiert permanent hektisch, und ein Ende ist vorerst nicht absehbar. Offenkundig setzt der Staat die Strafe nicht als ultima ratio ein, sondern immer öfters als bloss symbolisches Steuerungsmittel zur erhofften Bewältigung (oder pointierter: zur Ablenkung) von gesellschaftlichen Krisenphänomenen unterschiedlichster Art. $^{1}$ Wir bewegen uns da unaufhaltsam auf dem Weg «zu einem Interventionsrecht neuer Prägung» ${ }^{2}$ mit nebulösen Umrissen und einer Verwischung der traditionellen Grenze zwischen Repression und Prävention.

Der beschriebene Trend offenbart sich vorab in einem markanten Zuwachs von neuen Verboten und Geboten, die in rascher Kadenz entstehen. So manifestiert sich heute in unserer Gesellschaft (nicht zuletzt auch bei den Gerichten) ein uner-

Siehe Karl-Ludwig Kunz/Martino Mona, Rechtsphilosophie, Rechtstheorie, Rechtssoziologie, 2. Aufl., Bern 2015, Kp 7, Rn 67; ferner mit anschaulichen Beispielen aus der Gesetzgebungspraxis Marcel Alexander Niggli, Vom Repressions- zum Präventionsstrafrecht: Die Abkehr von der Ahndung begangener zur Verhinderung befürchteter Delikte, in: Strafverteidigung und Sicherheitswahn, hrsg. vom Forum Strafverteidigung, Wien 2014, S. $13 \mathrm{ff}$.

2 Niklaus Oberholzer, Verdeckte Massnahmen und Richtervorbehalt, forumpoenale 2011, S. 226. bittlicher Drang nach vergeltenden Reaktionen auf normwidriges Verhalten.

\section{b) Rechtsdogmatische Konsequenzen}

3 In rechtsdogmatischer Hinsicht findet beim gegenwärtigen Kriminalisierungsschub zumeist eine Regelungstechnik Anwendung, die durch weitgefasste Universalrechtsgüter in Verbindung mit abstrakten Deliktstatbeständen geprägt ist und zudem die Strafbarkeitsgrenze auf die Stufe von Vorbereitungshandlungen vorverlegt. Eine derartige Strategie bringt als Resultat eine flächendeckende Verbotsmaterie hervor, die auf Lückenlosigkeit abzielt. Solche Gesetzesbestimmungen entsprechen zumeist keinen materiellen Gerechtigkeitspostulaten. Neben der Bewältigung prozessualer Beweisprobleme 3 werden die neuen politischen Erwartungen an das Strafrecht erfüllt. Die zusätzlichen Verhaltensnormen fungieren demnach nicht nur als Basis für mehr Bestrafung, sondern ebenso als Anknüpfungspunkt für präventive Anliegen. ${ }^{4}$ Anstelle der begangenen Tat 5 bilden der böse Wille und dessen prognostizierte Verwirklichung den Angelpunkt der Bestrafung. Diese Ausdehnung der Strafbarkeitsgrenze soll den Behörden erlauben, möglichst frühzeitig einzugreifen. In den Vordergrund rückt der Blick in die Zukunft, und Bedürfnisse einer vorbeugenden Verbrechensbekämpfung sind angesprochen.

3 Grundlegend Detlef Krauss, Zur Funktion der Prozessdogmatik, in: Dogmatik und Praxis des Strafverfahrens, hrsg. von H. Jung/H. MüllerDietz, Köln etc. 1989, S. 9 f.

4 Zur Vertiefung des schillernden Begriffs «Prävention» hinten Ziff. III/2/c.

5 Vgl. Günter Stratenwerth, Schweizerisches Strafrecht, Band I, 4. Aufl., Bern 2011, § $12 \mathrm{~N}$ 3: «Sache des Strafrechts (...) ist allein die Reaktion auf den geschehenen Rechtsbruch.» 


\section{c) Mehr Sicherheit}

4 Eine derart zukunftsorientierte Ausrichtung gelangt sodann noch wesentlich stärker im Sanktionenrecht zur Geltung, wo namentlich bei schwerer Delinquenz das spezialpräventive Ziel der Rückfallverhinderung vorherrscht. Das zeigt sich insbesondere bei den therapeutischen und verwahrenden Massnahmen gemäss Art. 59 - 65 StGB. Diesbezüglich beeinflussen vor allem die geltend gemachten (realen oder vermeintlichen) Sicherheitsbedürfnisse der Bevölkerung die gesellschaftlichen Diskussionen und als Folge davon die Strafgesetzgebung. Dabei dominiert heute allerdings eine einseitige Sicherheitsoptik, die durch polizeiliches Denken genährt wird. Sie setzt in einem instrumentell-mechanischen Sinne vorwiegend auf Gefahrenabwehr, und zwar mit Mitteln des Strafrechts. Im Fokus stehen Einsperren und Isolieren auf unbestimmte Zeit.

Sobald die Sicherheit als Rechtsgut oder gar als Grundrecht mystifiziert und in den politischen Diskurs unreflektiert eingeführt wird, fördert das unvermeidbar masslose staatliche Interventionen. ${ }^{6}$ Gleichzeitig werden die verfassungsmässigen Freiheitsrechte der beschuldigten Personen einer Geringschätzung preisgegeben.7 Prävention und Gefahrenabwehr kennen eben keine immanenten Beschränkungen; das Prinzip der Verhältnismässigkeit muss vielmehr von aussen her eingeführt werden. Erschwerend

Siehe Günter Frankenberg, Kritik des Bekämpfungsrechts, KJ 2005, S. 375 f.; ferner Winfried Hassemer, Warum Strafe sein muss, Berlin 2009, S. $75 \mathrm{ff}$.

Instruktiv z.B. Bernhard Haffke, Vom Rechtsstaat zum Sicherheitsstaat?, KJ 2005, S. 20 und Erhard Denninger, Freiheit durch Sicherheit?, KJ 2002, S. $470 \mathrm{ff}$. kommt sodann noch hinzu, dass «Sicherheit» begrifflich kaum präzise definierbar ist und ausserdem ein nie (vollständig) erfüllbares Ideal darstellt. ${ }^{8}$ Wenn das Strafrecht überbordender Prävention entgegenwirken will, muss es auf seiner mässigenden Funktion beharren und darf sich nicht in die polizeiliche Gefahrenabwehr einspannen lassen. Diesbezüglich sei an das eindrucksvolle Votum von Winfried Hassemer erinnert:

«Während ein Gefahrenabwehrrecht die Herstellung von Sicherheit unmittelbar zu seinem Ziel macht, darf das Strafrecht Sicherheit gleichsam nur vermittelt herstellen: im Rücken gleichmässiger und angemessener Antwort auf das Verbrechen, im Rahmen freiheitswahrender und verhältnismässiger Reaktion. Das Strafrecht muss, wenn es sein Unrechtsurteil rechtfertigen will, der Person gerecht werden, der gegenüber dieses Urteil gefällt wird, und es darf diese Verpflichtung auf personale Angemessenheit und Gerechtigkeit seiner Eingriffe auch in Zeiten präventiver Weltverbesserung nicht verdrängen. Hoffnung auf und Bewerkstelligung von Prävention und Gefahrenabwehr sind im Strafrecht nur akzeptabel im Rahmen einer angemessenen Antwort auf Unrecht und Schuld.»9

8 Erhard Denninger, Prävention und Freiheit, in: Vom Rechtsstaat zum Präventionsstaat, hrsg. von S. Huster/K. Rudolph, Frankfurt a.M. 2008, S. 92 und $94 \mathrm{f}$.

9 Sicherheit durch Strafrecht, in: Jenseits des rechtsstaatlichen Strafrechts, hrsg. vom Institut für Kriminalwissenschaften und Rechtsphilosophie, Frankfurt a.M. 2007, S. 135; siehe ferner Ingeborg Zerbes, Spitzeln, Spähen, Spionieren, Wien/New York 2010, S. $244 \mathrm{f}$. 


\section{Der Wandel im Verfahren}

\section{a) Neue Schwerpunkte}

Die Expansion des materiellen Rechts kann nicht ohne Konsequenzen auf die Ausgestaltung des Strafverfahrens bleiben. Das grenzenlose Sicherheitsdenken beim Erlass von Strafnormen und sanktionen findet seine Fortsetzung in der Prozessordnung. Zunächst einmal fällt auch bei einer bloss oberflächlichen Betrachtung des Verfahrensrechts schon seit längerer Zeit eine unverkennbare Schwerpunktverlagerung von der richterlichen Hauptverhandlung zur Voruntersuchung auf. Das ist u.a. eine Folge der erheblichen Ausdehnung und Vorverlegung der Strafbarkeitsgrenzen im materiellen Recht. Daneben spielt auch die starke Eigendynamik in der technischen Entwicklung der (geheimen) Ermittlungsmethoden eine wesentliche Rolle; diese fördert zwangsläufig den polizeilichen Handlungsbedarf ${ }^{10}$. Die traditionell zentrale Aufgabe einer tatverdachtsbezogenen Wahrheits- und Rechtsfindung durch unabhängige Gerichte verliert zusehends an Gewicht. An Bedeutung gewinnt hingegen die Aufgabe einer umfassenden Informationsbeschaffung. Heute interessiert demnach mehr der Anfang als das Ende des Verfahrens.

7 Hinzu kommt sodann ein intensives Streben nach Beschleunigung der Prozesse. Als Mittel hierfür gelangen in erster Linie Vereinfachungen im Verfahrensablauf zum Einsatz, welche eine zeitliche Verkürzung erwarten lassen. Gefragt sind rasche und einfache «Lösun-

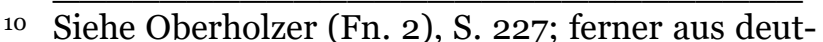
scher Sicht Mark A. Zöller, Heimliche und verdeckte Ermittlungsmassnahmen im Strafverfahren, ZStW 2012, S. 416. gen». Die Strenge gewisser prozessualer Formen wird gelockert, womit sich eine nicht unerhebliche Zeitersparnis verbindet. Auf Schritt und Tritt begegnet man in der Schweizerischen Strafprozessordnung Bestimmungen, welche massiv auf eine Beschleunigung abzielen (ganz besonders bspw. im Strafbefehlsverfahren gemäss Art. 352 ff. StPO ${ }^{11}$ ). ${ }^{12}$ Die Vereinfachung der Verfahrensabläufe ist geeignet, rascher ein Resultat $\mathrm{zu}$ erreichen und (im Falle eines Schuldspruchs) den staatlichen Strafanspruch zu realisieren. Die präventiven Anliegen des materiellen Rechts sollen möglichst frühzeitig Wirkung entfalten können. Demgegenüber kommt der Vergeltung einer vor Gericht sorgfältig ermittelten Tatschuld eine eher untergeordnete Bedeutung zu.

\section{b) Mehr Zwangsmassnahmen}

8 In ausgeprägter Weise offenbart sich das gewandelte Strafverfahren bei den zur Verfügung stehenden prozessualen Eingriffsmitteln. So stehen die «modernen, tendenziell flächendeckenden und überwiegend geheimen Zwangsmassnahmen»13 im Aufwind. Das lässt sich anschaulich daran ablesen, dass die Schweizerische Strafprozessordnung diesem Bereich weit mehr als hundert (!) Gesetzesbestimmungen widmet (Art. 196 - 298d StPO). Erwähnenswert sind etwa die klassischen freiheitsentziehenden Zwangsmassnahmen (Art. 215 ff. StPO), ausserdem aber auch etwa die Hausdurchsuchung (Art. 244 f. StPO) und die Beschlagnahme (Art. 263 ff. StPO). Darüber hinaus fällt die ungewöhnlich de-

11 Schweizerische Strafprozessordnung vom 5. Oktober 2007 (SR 312.0).

12 Vgl. dazu den Überblick bei Mark Pieth, Schweizerisches Strafprozessrecht, 3. Aufl., Basel 2016, S. 248 ff., mit zahlreichen Literaturangaben.

13 Pieth (Fn. 12), S. 134. 
taillierte Regelung von neu eingeführten verdeckten Massnahmen auf, beispielsweise die Überwachung des Post- und Fernmeldeverkehrs (Art. $269 \mathrm{ff}$. StPO) sowie die verdeckte Ermittlung und Fahndung (Art. 285a ff. und 298a ff. StPO). Diese quantitative wie strukturelle Erweiterung des polizeilichen Ermittlungsarsenals im Dunst der Heimlichkeit stellt erhöhte Anforderungen an die Legitimation. ${ }^{14}$

\section{c) Überwachung und Kontrolle}

9 Eine vertiefte Beschäftigung mit den beschriebenen prozessualen Entwicklungen offenbart, dass sich im Verfahren (wie erwähnt) die Grenzen zwischen Repression und Prävention verwischen. Die Strafverfolgungsbehörden kümmern sich nämlich in weiten Bereichen gegenwärtig tendenziell eher um die Aufdeckung (vermuteter) krimineller Strukturen als um die Tatvorwürfe gegenüber einzelnen Verdächtigen.15 Mit den polizeilichen Vorermittlungen soll vielfach ein konkreter Tatverdacht überhaupt erst begründet werden. Bezweckt wird hier «die Gewährleistung der Sicherheit, die Kontrolle über Personen und Räume insgesamt».16 In diesem Rahmen erleben die

14 Allgemein dazu Pieth (Fn. 12), S. 160 ff. und Sabine Gless, Heimliche Ermittlungsmassnahmen im Schweizer Strafprozess, ZStW 2012, S. 440 ff.

15 Siehe auch Oberholzer (Fn. 2), S. 228; im gleichen Sinne für Deutschland Edda Wesslau, Waffengleichheit mit dem «Organisierten Verbrechen»?, KritV 1997, S. 241.

16 Mark Pieth, Vom Inquisitionsprozess direkt zum postmodernen Kontrollmodell?, in: Festschrift für Stefan Trechsel, hrsg. von A. Donatsch/M. Forster/C. Schwarzenegger, Zürich 2002, S. 428; ebenso Anna Coninx/Martino Mona, Strafprozessualer Zwang und positive Schutzpflichten Verbrechensverhütung als Legitimation von Zwangsmassnahmen, ZStrR 2017, S. 1 f. - Ferner aus polizeilicher Sicht Christiane Lentjes Meili, Präventiv oder Repressiv?, in: Liber amicorum geheimen Überwachungsmassnahmen (Art. 269 ff. StPO) als «das wohl populärste Phänomen der strafrechtlichen Gefahrenabwehr» 17 einen imposanten Aufschwung.

10 Ganz generell hat der Strafprozess begonnen, sich $\mathrm{zu}$ verselbständigen und dem Polizeirecht anzunähern. Namentlich das herkömmliche Konzept strafrechtlicher Prävention ist ohne klar sichtbare Konturen ausgedehnt worden auf allgemeine polizeiliche Bedürfnisse der Informationsbeschaffung sowie der Überwachung und Kontrolle von Risiken. ${ }^{18}$.

\section{d) Präventive Verseuchung}

11 Die soeben skizzierten rechtspolitischen Schwerpunktverlagerungen lassen «starke Anzeichen einer präventiven Verseuchung des Prozessrechts» feststellen.19 Eine derart kritische Bemerkung mag vielleicht erstaunen; sie widerspiegelt jedenfalls ein manifestes Unbehagen gegenüber dem aktuellen Rechtszustand. Erkennbar wird «eine Funktionserweiterung des Strafprozesses - eine Aufrüstung zugunsten der Gefahrenabwehr, auch gerne etwas holprig als «Verpolizeilichung > beschrieben -, die zu einer Beschneidung traditionell eingriffsfreier Bereiche führt». ${ }^{20}$ Konkret stellt sich nun

für Andreas Donatsch, hrsg. von A. Cavallo u.a., Zürich/Basel/Genf 2012, S. 437 ff.

17 Zerbes (Fn. 9), S. 298.

18 Vgl. auch Hassemer (Fn. 6), S. 156 f. und Zerbes (Fn. 9), S. 297.

19 Peter Albrecht, Strafrecht ohne Recht?, ZStrR 2013, S. 392; zustimmend Coninx/Mona (Fn. 16), S. $1 \mathrm{f}$.

20 Ingeborg Zerbes, Geheime Überwachung im Strafprozess: Sicherheitsgefühl vor Freiheit?, in: Gedenkschrift für Edda Wesslau, hrsg. von F. Herzog/R. Schlothauer/W. Wohlers, Berlin 2016, S. 468, unter Hinweis auf Wesslau. 
die Frage, was die hervorgehobene «Seuche» inhaltlich bedeuten soll und inwieweit dadurch grundlegende Maximen eines rechtsstaatlichen Strafprozessrechts der Erosion ausgesetzt sind.

Der hier aufgeworfenen Thematik widmet sich der vorliegende Aufsatz anhand der heute so bedeutsamen (und teils innovativen) prozessualen Zwangsmassnahmen. Gerade in diesem Rechtsbereich sind die präventiven Wucherungen samt ihren drastischen Auswirkungen auf die individuellen Freiheitsrechte evident, und gleichzeitig befinden sich die davon betroffenen Personen aus prinzipiellen Gründen von vorneherein in einer geschwächten Position. ${ }^{21}$ Zum besseren Verständnis der angesprochenen juristischen Problematik bedarf es zunächst einer kurzen Einführung in der Form allgemeiner theoretischer Überlegungen zu den Funktionen des Prozessrechts (nachfolgend Ziff. II). Im Anschluss daran folgt eine weiterführende Analyse bestimmter präventiver Zwangsmassnahmen (nachfolgend Ziff. III und IV).

\section{Funktionen des Prozessrechts}

\section{Die Verwirklichung materiellen Rechts}

Dem Prozessrecht obliegt nach allgemeiner Ansicht als dienende Aufgabe die Verwirklichung des materiellen Strafrechts. ${ }^{22}$ Die Prozessordnung regelt die

21 Lehrreich dazu Coninx/Mona (Fn. 16), S. 5 ff.: «Schutzbedürfnisse der Mehrheit und Freiheitsrechte der Minderheit».

22 Siehe statt vieler Niklaus Schmid/Daniel Jositsch, Handbuch des schweizerischen Strafprozessrechts, 3. Aufl., Zürich/St. Gallen 2017, N 6. Im Übrigen gilt ebenso das Umgekehrte, was bedeutet, dass das materielle Recht durchaus auch der Verwirklichung des Prozessrechts «dient»
Organisation der Strafverfolgungsbehörden und schreibt vor, auf welche Weise die Strafbarkeit einer bestimmten Person im konkreten Fall zu ermitteln ist. Die materiell-rechtlichen Wertungen des Gesetzgebers bilden zunächst bloss (abstrakte) «Hülsen»,23 die durch entsprechende Ergänzungen auf der Verfahrensebene aufzufüllen sind. Erst dadurch nimmt das Strafrecht seine konkrete Gestalt und Geltung in der sozialen Realität an. ${ }^{24}$

14 Nach traditionellem Rechtsverständnis ist der Strafprozess retrospektiv ausgerichtet und befasst sich mit der durch rechtliche Normen geleiteten Aufarbeitung von verübten Straftaten (oder präziser: von mutmasslich begangenem Unrecht). Seine Optik ist demnach vergangenheitsorientiert, indem es um die Rekonstruktion eines abgeschlossenen Geschehens geht. 25 Bezweckt wird damit die Durchsetzung des staatlichen Strafanspruchs, also im Wesentlichen ein repressives Anliegen. Den Ausgangspunkt für die behördlichen Aktivitäten bildet der konkrete Tatverdacht, ${ }^{26}$ der sich in-

(zutreffend Krauss [Fn. 3], 3 ff.). Als ein für unseren Zusammenhang (siehe hinten Ziff. III/3) instruktives Beispiel sei das Urteil BGE 131 IV 100 erwähnt, wo die Bedürfnisse einer verdeckten Ermittlung den materiell-rechtlichen Entscheid über den Beginn eines strafbaren Versuchs in bedenklicher Weise steuerten (sehr kritisch dazu Peter Albrecht, Sexuelle Handlungen mit Kindern: Versuch oder Vorbereitung, AJP 2005, S. 751 ff., insb. S. 755 f.).

23 Winfried Hassemer, Einführung in die Grundlagen des Strafrechts, 2. Aufl., München 1990, S. 119 .

24 Siehe auch Niklaus Oberholzer, Grundzüge des Strafprozessrechts, 3. Aufl., Bern 2012, N 3; ferner ausführlich Klaus Ferdinand Gärditz, Strafprozess und Prävention, Tübingen 2003, S. 52 ff.

25 Vgl. Zerbes (Fn. 9), S. 244 ff. und Gärditz (Fn. 24), S. $60 \mathrm{ff}$.

26 Siehe auch BGE 140 I 353 E. 5.1 S. 360; weiterführend dazu sogleich Ziff. II/2/a. 
haltlich stets auf das materielle Recht, d.h. auf einen bestimmten Straftatbestand, zu beziehen hat. Ohne präzisen Blick auf die Verbotsmaterie in den Strafgesetzen wäre unklar, «wonach im Verfahren überhaupt geforscht, was überhaupt ermittelt werden soll». ${ }^{27}$

\section{Eigenständige Verfahrensinteressen}

\section{a) Schutz vor behördlichen Übergriffen}

Trotzdem: Eine derartige Fokussierung des Strafprozessrechts auf seine untergeordnete, dienende Rolle bleibt einseitig und oberflächlich. Dessen Funktionen sind nämlich wesentlich umfassender und beschränken sich nicht auf «die Verlängerung des materiellen Strafrechts in die Wirklichkeit, in einen Fall».28 Vielmehr handelt es sich beim Verfahrensrecht um ein Rechtsgebiet, das über seine dienende Aufgabe hinaus zusätzlich eigenständige Interessen und Ziele verfolgt. Dazu gehört u.a. der Schutz der in das Verfahren involvierten Personen vor behördlicher Willkür. ${ }^{29}$ Vor diesem Hintergrund versteht sich beispielsweise das Erfordernis eines sog. Anfangsverdachts für die Einleitung von Strafuntersuchungen, dies als ein aus dem Prinzip der Unschuldsvermutung (Art.10 Abs. 1 StPO) abgeleiteter Schutzfaktor. Der konkrete Tatverdacht löst einen sozialen Konflikt aus, der durch Aufklärung zu beseitigen ist. Daran haben sich die staatlichen Ermittlungsbemühungen $\mathrm{zu}$ orientieren. Man spricht hier vom Gebot der Ver-

27 Kurt Seelmann/Christopher Geth, Strafrecht, Allgemeiner Teil, 6. Aufl., Basel 2016, N 47.

28 Hassemer (Fn. 23), S. 119.

29 Siehe Oberholzer (Fn. 24), N 9; vgl. auch Gärditz (Fn. 24), S. $73 \mathrm{ff}$. dachtssteuerung der Behördentätigkeit. Dieses Gebot bezweckt, «den Beschuldigten und unverdächtige Dritte vor staatlicher Ermittlungswillkür zu schützen. Ohne Anfangsverdacht oder allmählich losgelöst von einem anfänglich bestehenden Tatverdacht unspezifisch nach Verdachtsmomenten und Beweismitteln $\mathrm{zu}$ 〈fischen〉 ist den Untersuchungsbehörden verboten.»30

16 Abgesehen davon gilt für die behördliche Ermittlungstätigkeit gemäss Art. 6 StPO der elementare einschränkende Grundsatz, dass das angestrebte Ziel nicht alle Mittel heiligt. Vielmehr darf die Erforschung der Wahrheit nicht um jeden Preis erfolgen, ${ }^{31}$ was beispielsweise in der Statuierung von Beweisverboten nach Art. 140 und 141 StPO erkennbar wird. Solche Regelungen sind ebenfalls unmittelbar auf den Schutz der betroffenen Individuen ausgerichtet.

\section{b) Justizförmigkeit des Verfahrens}

17 Soweit das Prozessrecht diese ihm obliegenden Schutzaufgaben übernimmt und wirksam erfüllt, darf man es als angewandtes Verfassungsrecht bezeichnen. Mit der Orientierung an der Verfassung verbindet sich das Gebot der Justizförmigkeit des Verfahrens. Dies ist eine Konsequenz aus der jedem Prozess inhärenten Gefahr eines Missbrauchs staatlicher Macht. Die Durchsetzung des Strafanspruchs hat demnach gemäss einem

$30 \overline{\text { Judith Natterer, Die Verwertbarkeit von Zufalls- }}$ funden aus der Telefonüberwachung im Strafverfahren, Bern 2001, S. 3.

31 In diesem Sinne statt vieler z.B. Wolfgang Wohlers, Kommentar zur Schweizerischen Strafprozessordnung (StPO), hrsg. von A. Donatsch/ Th. Hansjakob/V. Lieber, 2. Aufl., Zürich/Basel/ Genf 2014, Art. 6 N 2. 
durch das Gesetz genau beschriebenen Ablauf stattzufinden (Art. 2 Abs. 2 StPO).

Die geforderte Justizförmigkeit stellt einen bewusst eingerichteten Gegenpol zur Effizienz des Strafverfahrens dar. Sie dient «nicht nur dem Schutz des Individuums, in ihr kommt auch das rechtsstaatliche Gemeininteresse an der Begrenzung staatlicher Macht zum Ausdruck». ${ }^{2}$ Man spricht im vorliegenden Zusammenhang ebenfalls von den schützenden Formen des Prozessrechts. Solchermassen wird «zum Ausdruck gebracht, dass die Förmlichkeiten des Strafverfahrens keine Formsache sind».33 Sie bilden keinen Selbstzweck, sondern schützen verfassungsmässige Grundrechte. Das sollte man sich gerade in der Praxis immer wieder vergegenwärtigen, obwohl die prozessuale Formenstrenge den Verfahrensablauf stört und Zeit beansprucht. 34

\section{Präventive Zwangsmassnahmen im Strafprozess}

\section{Eine Klage aus der Wissenschaft}

19 In jüngster Zeit beklagen sich einzelne Vertreter der Rechtswissenschaft:

«Zwangsmassnahmen im Strafprozessrecht werden zunehmend als Instrument zur Verbrechensprävention eingesetzt und orientieren sich entsprechend am Konzept der Gefährlichkeit und der Risikoabwehr. Dabei geht es nur noch am Rande um Wahrheitsfindung und Auf-

Pieth (Fn. 12), S. 3.

33 Hassemer (Fn. 23), S. 136.

34 Allgemein zur gebotenen «Bremse des Verfahrens» Niklaus Ruckstuhl/Volker Dittmann/Jörg Arnold, Strafprozessrecht, Zürich/Basel/Genf 2011, N $110 \mathrm{ff}$. klärung einer Straftat, die in eine gerichtliche Entscheidung - eine Verurteilung oder einen Freispruch - mündet. Stattdessen wird auch das Strafprozessrecht durch das Dogma der <Verbrechensverhütung um jeden Preis vereinnahmt und entpuppt sich als belastbarer Aufhänger für eine Reihe von einschneidenden präventiven Massnahmen.»35

20 Die zitierte Klage gibt die Situation in der Praxis zutreffend wieder und bedarf deshalb im Folgenden einer näheren Erläuterung. Zur Illustration hierfür werden zwei Beispiele herangezogen, die zu Eingriffen in die Freiheitsrechte der beschuldigten Personen führen und überdies präventiv durchdrungen sind. Es handelt sich einerseits um die präventive Untersuchungshaft (Ziff. III/2) und andererseits um die präventive verdeckte Polizeitätigkeit (Ziff. III/3).

\section{Die präventive Untersuchungshaft}

\section{a) Zur Legitimation von Untersuchungshaft}

21 Die Berechtigung für die Anordnung einer Untersuchungshaft steht unter hohen Anforderungen. Denn es handelt sich dabei um einen schwerwiegenden Freiheitsentzug, der Personen betrifft, die (noch) nicht verurteilt sind und deshalb von Rechts wegen als unschuldig gelten (Art. 10 Abs. 1 StPO, Art. 32 Abs. 1 BV und Art. 6 Ziff. 2 EMRK). Der Gesetzgeber bemühte sich, den hier angedeuteten Konflikt mit der Unschuldsvermutung zu beseitigen (oder wenigstens zu entschärfen), indem er für eine solche Zwangsmassnahme den dringenden Verdacht voraussetzt, ein bestimmtes Delikt verübt

$35 \overline{\text { Coninx/Mona (Fn. 16), S. } 1 .}$ 
zu haben (Art. 221 Abs. 1 StPO). ${ }^{6}$ Der Tatverdacht bedeutet eine dem Angeschuldigten zurechenbare Störung der Rechtsordnung. Die störende Wirkung dauert an, solange der Verdacht nicht entkräftet ist oder auf die begangene Tat keine staatliche Reaktion folgt. Daraus rechtfertigt es sich, den Verdächtigen als «Störer» zur Aufklärung des vorhandenen Verdachtes hoheitlich in Anspruch zu nehmen,37 und zwar allenfalls auch mittels Untersuchungshaft. Auf dieser Basis finden in einem auf Wahrheitssuche verpflichteten Strafverfahren (Art. 6 i.V.m. Art. 196 StPO) die beiden klassischen Haftgründe der Flucht- und der Verdunkelungsgefahr ihre prinzipielle Berechtigung (Art. 221 Abs. 1 lit. a und b StPO).

\section{b) Präventive Haftgründe}

Wesentlich anders verhält es sich hingegen bei denjenigen gesetzlichen Haftgründen, die nicht (primär) die Sicherung eines laufenden Verfahrens im Visier haben, sondern vielmehr eine Verhinderung künftiger Straftaten. Für eine solche (verfahrensfremde) Inhaftierungsmöglichkeit wird argumentativ zuweilen auf die relativen Strafzwecke verwiesen und daraus die Berechtigung der Spezialprävention als Haftgrund abgeleitet. ${ }^{8}$ Solchermassen sind «Ziele des ma-

$36 \overline{\text { Ausführlich zur theoretischen Begründung Detlef }}$ Krauss, Der Grundsatz der Unschuldsvermutung im Strafverfahren, in: Strafrechtsdogmatik und Kriminalpolitik, hrsg. von H. Müller-Dietz, Köln etc. 1971, S. 167 ff.; vgl. auch Fabio Manfrin, Ersatzmassnahmenrecht zur Schweizerischen Strafprozessordnung, Zürich/Basel/Genf 2014, S. $24 \mathrm{ff}$.

37 Zerbes (Fn. 9), 244.

38 Ulrich Weder, Die gefährliche beschuldigte Person und die Wiederholungs- und Ausführungsgefahr, ZStrR 2014, S. 368 f.; ferner BGE 143 IV 9 E. 2.2 S. 11 . teriellen Strafrechts in das formelle Strafrecht eingewandert und haben sich alsbald breitgemacht».39 Zur Beschwichtigung wird oft betont, dass Art. 5 Ziff. 1 lit. c EMRK ausdrücklich die Notwendigkeit anerkenne, Beschuldigte an der Begehung strafbarer Handlungen zu hindern.40 Das genügt indessen nicht, um die Präventivhaft zu legitimieren; denn die Vereinbarkeit einer bestimmten Gesetzesbestimmung mit den europäischen Mindeststandards bedeutet nicht automatisch, dass die betreffende Norm auch sachgerecht und rechtsstaatlich akzeptabel ist. Dies gilt hier umso mehr, als die Möglichkeiten einer präventiven Inhaftierung nach der Strassburger Rechtsprechung zur zitierten EMRK-Norm äussert limitiert sind 41 .

23 Für die Erweiterung des Haftrechts werden sodann auch positive Schutzpflichten des Staates hervorgehoben, welche sich aus dem verfassungs- und völkerrechtlich geschützten Recht auf Leben ergeben ${ }^{4}$. So betont denn das Bundesgericht ausdrücklich die staatliche Pflicht, «präventiv Schutzmassnahmen zu ergreifen, wenn das Leben einer Person durch Dritte bedroht wird». Falls «von kriminellen Handlungen eines Dritten reell und unmittelbar eine derartige Gefahr ausgeht», seien die Behörden "verpflichtet, die in ihrer Macht stehenden geeigneten Mass-

$39 \overline{\text { In diesem Sinne mit Blick auf das deutsche Recht }}$ kritisch Hassemer (Fn. 9), S. 109 f.

40 BGE 143 IV 9 E. 2.2 S. 11 und 137 IV 84 E. 3.2 S. 85 .

41 Siehe Anna Coninx, Haft wegen Ausführungsgefahr - Notwendige Beschränkung einer hybriden Rechtsfigur zwischen Strafprozessrecht und Polizeirecht, ZSR 2016 I, S. 387 f. und Adrian Dumitrescu, Die Präventivhaft nach Art. 221 Abs. 2 StPO, AJP 2015, S. 449 ff.

42 Weder (Fn. 38), S. 369 f. und Dumitrescu (Fn. 41), S. 447 f. 
nahmen zu ergreifen (...)».43 Allerdings fehlt hier eine nähere Erklärung, wo im uferlosen Vorfeld eines konkreten Tatverdachts die Grenzen des erwähnten Schutzes zu ziehen sind und weshalb er gerade mittels Haftanordnung im Strafprozess erfolgen soll. Schwer wiegt diesbezüglich insbesondere die Befürchtung, dass längerfristig eine «Verwischung der Grenzen zwischen formellem und materiellem Strafrecht» stattfinden und diese «zu Lasten verfahrensrechtlicher Strenge und Zurückhaltung» gehen wird.44 Somit bleibt es dabei, dass die beschriebene präventive Zielsetzung den dargelegten Grundsätzen einer Legitimation von Untersuchungshaft widerspricht. 45

\section{aa) Fortsetzungs- oder Wiederholungsgefahr}

24 Zunächst sei einmal auf Art. 221 Abs. 1 lit. c StPO verwiesen, wonach Untersuchungshaft gegen eine dringend beschuldigte Person zulässig ist, «wenn ernsthaft zu befürchten ist, dass sie durch schwere Verbrechen die Sicherheit anderer erheblich gefährdet, nachdem sie bereits früher gleichartige Straftaten verübt hat». Dieser Haftgrund versteht sich - gemäss der Intention der bundesrätlichen Botschaft - ausschliesslich als Gefahrenabwehr; es handle «sich somit eigentlich um eine sichernde, polizeiliche Zwangsmassnahme». 46

43 BGE 139 IV 121 E. 4.6 S. 125 f., unter Hinweis auf die Rechtsprechung des EGMR. - Grundlegend zur Problematik der positiven Schutzpflichten im Rahmen der prozessualen Zwangsmassnahmen Coninx/Mona (Fn. 16), S. 3 ff.

44 Hassemer (Fn. 9), S. 111.

45 Siehe Hans-Ullrich Paeffgen, in: Systematischer Kommentar zur Strafprozessordnung, hrsg. von J. Wolter, 5. Aufl., Köln 2016, Rn 12 vor $\S \S 112$ ff.

46 Botschaft zur Vereinheitlichung des Strafprozessrechts vom 21. Dezember 2005 (BBl 2006 1085), S. 1229.

25 Eine solche Inhaftierung bildet jedoch einen dogmatischen Fremdkörper47 innerhalb des Prozessrechts und steht im Widerspruch zur Unschuldsvermutung. Denn die Präventivhaft bezweckt keine Sicherung der Strafuntersuchung. In der bundesgerichtlichen Rechtsprechung ist zwar schon mehrfach der Versuch unternommen worden, die Wiederholungsgefahr als Haftgrund mit Verfahrensargumenten zu «retten», nämlich mit der Erwägung, dass diese Haft dem prozessualen Ziel der Verfahrensbeschleunigung diene. ${ }^{8}$ Das Risiko einer blossen Verfahrensverzögerung oder -erschwerung vermag indessen niemals den Eingriff einer Untersuchungshaft in die persönliche Freiheit einer beschuldigten Person zu rechtfertigen.49 Abgesehen davon ist Art. 221 Abs. 1 lit. c StPO, wie sich aus dem Gesetzestext ergibt, ausschliesslich als Präventivhaft konzipiert, was auch dem Willen des Gesetzgebers entspricht.

26 (wenigstens prima vista) der Problematik des hier besprochenen Haftgrundes nicht ganz zu verschliessen, wenn es in seinen allgemeinen Erwägungen regelmässig eine restriktive Gesetzesanwendung anmahnt.50 Allerdings zeigt eine detaillierte Analyse der neuesten Praxis leider unmissverständlich, dass trotz verbaler höchstrichterlicher Beteuerungen von einer Zurückhaltung bei der Annahme einer Wiederholungsgefahr im Sinne des Gesetzes keine Rede sein kann. Vielmehr

47 Pieth (Fn. 12), S. 143.

48 Z.B. BGE 143 IV 9 E. 2.2 S. 11; BGE 137 IV 84 E. 3.2 S. 85; BGE 135 I 71 E. 2.2 S. 72.

49 Ebenfalls skeptisch Niklaus Schmid, Zum Haftgrund der Wiederholungs- und Fortsetzungsgefahr in der neueren schweizerischen Strafprozessentwicklung, SJZ 1987, S. 228.

50 Z.B. BGE 143 IV 9 E. 2.2 S. 11 f.; BGE 137 IV 84 E. 3.2 S. 85; BGE 135 I 71 E. 2.3 S. 73. 
hat der Anwendungsbereich von Art. 221 Abs. 1 lit. c StPO in einer sehr problematischen Weise eine permanente Erweiterung über den Wortlaut der Norm hinaus erfahren, ${ }^{1}$ was in der Literatur teilweise auf mehr oder minder deutliche Ablehnung gestossen ist. ${ }^{2}$ Als völlig inakzeptabel erweist sich, dass das Bundesgericht53 ausnahmsweise sogar das im Gesetz ausdrücklich verankerte Vortatenerfordernis - mittels «einer systematischteleologischen Auslegung» - weginterpretiert. Angesichts solcher Entwicklungen muss die unermüdlich repetitive Proklamation (in Urteilserwägungen), dass die Präventivhaft nur als «ultima ratio» zur Anwendung gelangen dürfe,54 für die (gleichwohl) Inhaftierten ziemlich zynisch klingen.

\section{bb)Ausführungsgefahr}

27 Noch einen Schritt weitergehend lockert der Art. 221 Abs. 2 StPO das Band zur dogmatischen Basis des Strafprozessrechts zusätzlich. Gestützt auf diese Bestimmung kommt Haftanordnung nämlich auch ohne jeglichen Tatverdacht in Betracht,55 «wenn ernsthaft zu befürchten ist, eine Person werde ihre Drohung, ein schweres Verbrechen auszuführen, wahrmachen». Zur Erläuterung führt die bundesrätliche Botschaft aus:

51 Ausführlich dazu BGE 143 IV 9 E. 2.3 ff. S. 12 ff. mit weiteren Hinweisen.

52 Siehe etwa Andreas Eicker, Zur bundesgerichtlichen Interpretation des Haftrechts contra legem, in: Festschrift für Martin Killias, hrsg. von A. Kuhn u.a., Bern 2013, S. 980 ff.; Manfrin (Fn. 36), S. 42 ff. und 151 ff.; Andreas Donatsch/Eliane Hiestand, Wortlaut des Gesetzes oder allgemeine Rechtsprinzipien bei der Auslegung der StPO, ZStrR 2014, S. 10 ff.

53 BGE 137 IV 13 E. 3 f. S. 18 ff.; ebenso BGE 143 IV 9 E. 2.3.1 S. 13 .

54 Statt vieler BGE 143 IV 9 E. 2.2 S. 12.

55 BGE 140 IV 19 E. 2.1.1 S. 21.
«Der Haft wegen Ausführungsgefahr liegt die Konstellation zugrunde, dass die Begehung eines schweren Verbrechens erst in Aussicht gestellt wird, der Bezug zu einer bereits begangenen Straftat indes fehlt, weshalb hier kein dringender Tatverdacht vorausgesetzt werden kann. Verlangt ist deshalb vielmehr eine konkrete Gefahr der Tatbegehung (〈ernsthaft zu befürchten〉). Mangels Anknüpfung an eine mutmasslich begangene Straftat ist auch weder von <beschuldigter Person> noch von <Untersuchungshaft> die Rede.»56

28 Die primäre Zielsetzung der Inhaftierung liegt hier offenkundig «im Schutz eines potentiellen Opfers vor einem schweren Verbrechen».57 Nach der Ansicht des Bundesgerichts kann die vom Gesetz verlangte Drohung auch konkludent erfolgen.58 Währendem dieser ausgeprägt präventive Haftgrund dem heutigen kriminalpolitischen Mainstream entsprechend in der Praxis zumeist ohne Skrupel Anerkennung findet, weisen doch einzelne Stimmen in der Literatur zu Recht auf dessen System- und Verfassungswidrigkeit hin. 59

\section{c) Prävention zwischen dem Straf- und dem Verwaltungsrecht}

\section{aa) Spezialprävention}

29 Wenn die gesetzlichen Bestimmungen mittels der Inhaftierung eine Verhinderung von (weiteren) Straftaten anstreben, mag man darin - in Anlehnung an das

56 Botschaft (BBl 2006, Fn. 46), S. 1229.

57 Siehe Coninx/Mona (Fn. 16), S. 2.

58 BGE 137 IV 339 E. 2.4 S. 340.

59 Ein Verstoss gegen die Verfassung liegt in der fehlenden Kompetenz des Bundes zum Erlass von Polizeigesetzen; siehe Pieth (Fn. 12), S. 145, mit Literaturhinweisen. 
Sanktionenrecht - eine spezialpräventive Zielsetzung erkennen. ${ }^{60}$ Eine solche Betrachtungsweise passt jedoch nicht zum Haftrecht im Strafprozess; sie verstösst gegen die Unschuldsvermutung. Denn die Beseitigung einer Rückfallgefahr als spezialpräventives Anliegen obliegt den Strafsanktionen und setzt somit ein (rechtskräftiges) Urteil voraus. ${ }^{61}$ Abgesehen davon widerspricht die Rückfallverhinderung durch Untersuchungshaft auch inhaltlich dem aus den Straftheorien bekannten Konzept der Spezialprävention. Bei der prozessualen Inhaftierung erfolgt der Schutz der Gesellschaft vor (neuen) Taten nämlich sehr einseitig mechanisch durch Wegsperren und Isolieren. Demgegenüber wird die vom materiellen Recht intendierte Resozialisierung offenkundig ausgeblendet. ${ }^{62}$

\section{bb)Gefahrenabwehr}

30 Unter den gegebenen Umständen liegt es näher, die präventiven Haftgründe als Mittel der «Gefahrenabwehr» zu deuten und darin «eine sichernde, polizeiliche Zwangsmassnahme» zu sehen. ${ }^{63}$ Eine solche Form der Prävention wird üblicherweise «ausgelöst, sobald ein durch die Rechtsordnung geschützter Wert akut bedroht ist». ${ }^{64}$ Es geht um «Eingrif-

60 Nachweise vorne in Fn. 38.

61 Im gleichen Sinne Martin Seelmann, Präventive Strafverfolgung - ein notwendiges Oxymoron?, in: Prävention und freiheitliche Rechtsordnung, hrsg. von A. Coninx/G. Ege/J. Mausbach, Zü̈rich/St. Gallen 2017, S. 113; Matthias Härri, Auswirkungen der Unschuldsvermutung auf das Recht der Untersuchungshaft, AJP 2006, S. 1219; Manfrin (Fn. 36), S. 142 f.

62 Ähnlich auch die Kritik von Härri (Fn. 61), S. 1219.

63 So zur Fortsetzungs- oder Wiederholungsgefahr Botschaft (BBl 2006, Fn. 46), S. 1229 und BGE 143 IV 9 E. 2.2 S. 12; ferner zur Ausführungsgefahr z.B. Pieth (Fn. 12), S. 145.

64 Zerbes (Fn. 9), S. 243. fe in individuelle Rechte zur Abwehr unmittelbar bevorstehender Gefahren». 65 An einer derart konsequent polizeilichen Sicherheitsoptik mangelt es freilich in der Ausgestaltung der genannten Haftgründe. Im Haftrecht fehlt nämlich das Erfordernis einer akuten Bedrohung, ${ }^{66}$ die rasch zu beseitigen wäre. Die behördliche Verhinderung künftiger Delikte ist im Strafverfahren vielmehr längerfristig angelegt, was sich allein schon in der möglichen Dauer der Untersuchungshaft zeigt, die sich diesbezüglich krass vom nur kurzfristig zulässigen Polizeigewahrsam unterscheidet.67 Dies ist eine Folge daraus, dass mit der präventiven Haftbefugnis Möglichkeiten einer polizeilichen Gefahrenabwehr der Strafprozessordnung aufgepfropft und strafrechtlich eingefärbt wurden. Das betrifft sowohl den Art. 221 Abs. 1 lit. c wie auch den Art. 221 Abs. 2 StPO.

31 Den skizzierten systematischen Konflikt hat Anna Coninx vor kurzem präzis und zutreffend herausgearbeitet:

«Haft wegen Ausführungsgefahr nach Art. 221 Abs. 2 StPO zeichnet sich durch einen hybriden Charakter aus. Auf einen polizeilich relevanten Zustand (Gefahr eines schweren Verbrechens) wird mit strafprozessrechtlichen Möglichkeiten eines Freiheitsentzugs reagiert. Polizeiliche und strafprozessuale Möglichkeiten des Freiheitsentzugs werden also kombiniert und dadurch in ihrem Ausmass, ihrem Anwendungsbereich und ihrer Wirkung potenziert. Was die Anordnungsvoraussetzungen angeht, wird der

65 Zerbes (Fn. 9), S. 244.

66 Immerhin verlangt aber Dumitrescu (Fn. 41), S. 452 und 454, für Art. 221 Abs. 2 eine unmittelbare Gefahr der Tatausführung.

67 Anschaulich Coninx (Fn. 41), S. $391 \mathrm{ff}$. 
Logik des Polizeirechts gefolgt, hinsichtlich der Intensität des Freiheitsentzugs insbesondere dem wichtigen Aspekt der Dauer - finden dagegen strafprozessuale Regelungen Anwendung. Der Gesetzgeber hat also aus zwei unterschiedlichen Rechtsdogmatiken Elemente herausgelöst und zu einer neuartigen Norm zusammengefügt und gebündelt. Diese Kombination von Polizeirecht und Strafprozessrecht erlaubt einen maximalen und tendenziell überschiessenden staatlichen Zugriff und ist deshalb problematisch.» 68

\section{d) Feststellung der Gefährlichkeit}

\section{aa) Normative Massstäbe}

Für die Annahme der präventiven Haftgründe ist - trotz unterschiedlicher Formulierungen im Gesetz - stets das Risiko künftiger Straftaten der betroffenen Personen von zentraler Relevanz. Da stellt sich dann jeweils die Frage, welche normativen Kriterien den geforderten Prognosen zugrunde zu legen sind. Konkret geht es um die notwendige Qualität der Tätergefährlichkeit. Der Gesetzwortlaut ist ziemlich vage gehalten und teilweise auch unklar ausgefallen. Deshalb überrascht denn auch nicht, dass zu diesen Haftgründen bereits eine reichhaltige, insgesamt aber wenig konsistente Gerichtspraxis existiert 69 .

Die Annahme des Haftgrundes der Ausführungsgefahr erfordert gemäss höchstrichterlicher Praxis hinsichtlich schwerer Verbrechen «eine sehr ungünstige Prog-

68 Coninx (Fn. 41), S. 388.

69 Vgl. die zahlreichen Hinweise bei Schmid/ Jositsch (Fn. 22), N 1024 ff. nose». $7^{\circ}$ Von dieser restriktiven Richtlinie weicht das Bundesgericht in gewissen Situationen freilich ab: Für den Fall «einer zu befürchtenden vorsätzlichen Tötung darf an die Annahme der Ausführungsgefahr kein allzu hoher Massstab angelegt werden. Anders zu entscheiden hiesse, potenzielle Opfer einem nicht verantwortbaren Risiko auszusetzen (...)».71 Auf ähnliche Weise ist auch die ursprünglich zurückhaltende Rechtsprechung zu Art. 221 Abs. 1 lit. c StPO inzwischen deutlich relativiert worden. So müsse bei Straftaten gegenüber speziell schutzbedürftigen Personengruppen (namentlich Kindern) aus Gründen des Opferschutzes ein strenger Massstab gelten ${ }^{72}$. Diese Entwicklungen belegen, in welchem Ausmass die (unabhängigen?) Gerichte hier auf politische Einflüsse reagieren und dementsprechend die Abs. 1 lit. c und Abs. 2 des Art. 221 StPO unbekümmert immer extensiver interpretieren.

\section{bb)Prognoseschwierigkeiten}

34 Zusätzlich ins Gewicht fallen die praktischen Schwierigkeiten, in dem unter Zeitdruck stehenden summarischen Haftverfahren (Art. 224 ff. StPO) zuverlässige Aussagen hinsichtlich künftiger Delinquenz zu machen. Den Zwangsmassnahmengerichten stehen hierfür in

$7 0 \longdiv { \text { BGE } 1 4 0 \text { IV } 1 9 \text { E. 2.1.1 S. 22; ebenso Urteil des } }$ Bundesgerichts 1B_281/2016 vom 5. September 2016, E. 4.1.

71 Urteil des Bundesgerichts 6B_990/2013 vom 10. Juni 2014, E. 2.3., mit Praxishinweisen; ebenso Markus Hug/Alexandra Scheidegger, in: Kommentar zur Schweizerischen Strafprozessordnung (StPO), hrsg. von A. Donatsch/Th. Hansjakob/V. Lieber, 2. Aufl., Zürich/Basel/Genf 2014, Art. 221 N 44; kritisch hingegen Coninx (Fn. 41), S. 394 f. sowie Coninx/Mona (Fn. 16), S. $8 \mathrm{f}$. und $11 \mathrm{f}$.

72 Ausführlich BGE 143 IV 9 E. 3 ff. S. 17 ff. 
der Regel keine forensischen Gutachten zur Verfügung, weshalb die Rückfallprognosen häufig auf einer sehr dünnen Tatsachenbasis beruhen.73 Deshalb fragt sich (namentlich bei Ersttätern), ob die Richterinnen und Richter nicht jeweils überfordert sind, allein aufgrund der Aktenlage mit hinreichender Gewissheit einen präventiven Haftgrund zu bejahen. 74 Unter den gegebenen Umständen können sie wohl nicht mehr leisten als «biedere Laienpsychologie», 75 womit sich unvermeidbar ein erhebliches Mass an Fehleinschätzungen verbindet. 76

Aufschlussreich ist hier, auf welche Weise das Bundesgericht die normativ wie praktisch anspruchsvolle Prognoseproblematik umschifft und weitgehend bagatellisiert:

«Die Einholung eines psychiatrischen Gutachtens ist zur Beurteilung der Rückfallgefahr nicht in jedem Fall notwendig (...). Erscheint ein solches im konkreten Fall erforderlich oder wurde es bereits in Auftrag gegeben, rechtfertigt sich die Aufrechterhaltung der Haft bei gemäss Aktenlage ungünstiger Prognose jedenfalls so lange, bis die Wiederholungsgefahr gutachterlich abgeklärt ist (...). Mit Blick auf das in Haftsachen geltende Beschleunigungsgebot kann insoweit die Einholung eines Kurz- oder Vorabgut-

73 Ebenso Manfrin (Fn. 36), S. 147 und 150 f.

74 Das gilt erst recht, falls man erhöhte Anforderungen an die Gefahr stellt, etwa eine Unmittelbarkeit derselben (vorne Fn. 66) oder eine sehr ungünstige Prognose (vorne Ziff. III/2/d/aa) fordert.

75 Paeffgen (Fn. 45), § 112a, Rn 9, der hier etwas polemisch von «Prognosen» spricht, «die zumeist nur wenig von Kaffeesatzleserei entfernt sind».

76 Allgemein zu den hohen Fehlerquoten der Kriminalprognosen und zur Überschätzung der Rückfallgefahr Karl-Ludwig Kunz/Tobias Singelnstein, Kriminologie, 7. Aufl., Bern 2016, § 21, Rn 27 ff. achtens beim beauftragten Sachverständigen zur Frage der Rückfallgefahr angezeigt sein (...).»77

36 Solche Erwägungen lassen Zweifel darüber auftauchen, wie ernst es die Gerichte mit den vom Gesetz verlangten Prognosen wirklich nehmen. Wann die Einholung einer psychiatrischen Expertise überhaupt erforderlich ist, lässt das Bundesgericht nämlich vollkommen offen. ${ }^{78}$ Ausserdem gewinnt man leicht den Eindruck, dass es für den Haftentscheid eigentlich gar nicht so sehr auf eine fundierte individuelle Prognose ankommt. So ist eine Inhaftierung wegen Ausführungsgefahr (Art. 221 Abs. 2 StPO) u.U. selbst dann zulässig, "wenn die vorhandenen Fakten keine genaue Risikoeinschätzung erlauben».79 Demnach darf die notwendige Gefahr künftiger Delinquenz ohne Weiteres angenommen werden, solange diese nicht durch eine gutachterliche Stellungnahme «widerlegt» ist ${ }^{80}$. Abgesehen davon besteht bei der Wiederholungsgefahr nach Art. 221 Abs. 1 lit. c StPO zuweilen eine gewisse Neigung,

77 BGE 143 IV 9 E. 2.8 S. 16 f. betr. Wiederholungsgefahr.

78 Im Übrigen wäre es gewiss lohnend, die richterliche Auseinandersetzung mit (im Verfahren bereits vorliegenden) Gutachten bzw. Vor- oder Kurzgutachten einmal vertieft zu überprüfen. Mir erscheint es jedenfalls, dass das Bundesgericht tendenziell stark bestrebt ist, aus den psychiatrischen Stellungnahmen ungünstige Prognosen herauszulesen: illustrativ z.B. BGE 143 IV 9 E. 3.3 ff. S. 18 ff. und Urteil des Bundesgerichts 1B_487/2017 vom 1. Dezember 2017, E. 3.6 ff.

79 BGE 140 IV 19 E. 2.1.1 S. 22 und Urteil des Bundesgerichts 6B_990/2013 vom 10. Juni 2014, E. 2.3; kritisch dazu Coninx/Mona (Fn. 16), S. 11 f.; Coninx (Fn. 41), S. 391; Seelmann (Fn. 61), S. 119 .

80 So z.B. im Urteil des Bundesgerichts 6B_990/2013 vom 10. Juni 2014, E. 2.4.4, betr. Art. 221 Abs. 2; vgl. ausserdem das Urteil des Bundesgerichts 1B_174/2013 vom 27. Mai 2013, E. 3.6. 
vorwiegend abstrakt - etwa aus der Art der Delinquenz - auf eine erhebliche Gefährdung der Sicherheit zu schliessen. ${ }^{81}$

\section{cc) Anschein der Gefährlichkeit}

Der vertiefte Blick in die gesetzgeberische Ausgestaltung und die richterliche Anwendung der präventiven Haftgründe führt zur Einsicht, dass die Gerichtspraxis vom Erfordernis einer Individualprognose immer wieder abweicht. Anstelle einer möglichst präzisen Aussage über die allenfalls zu erwartende künftige Delinquenz der beschuldigten Person begnügt man sich nicht selten mit dem erkennbaren Anschein einer Gefährlichkeit. Es geht der Sache nach jeweils um die normative Zuordnung zu einer Risikogruppe. Im Fokus steht dabei vor allem eine dezidierte - teils dramatisierte - Bewertung der Schwere der Straftaten, mit denen zu rechnen ist. Der Grad der im Einzelfall sehr schwierig zu ermittelnden Wahrscheinlichkeit deliktischen Verhaltens spielt hingegen eine untergeordnete Rolle. ${ }^{82}$ Insoweit verfolgt also die Inhaftierung nicht wirklich primär eine polizeiliche Gefahrenabwehr, sondern erscheint eher als symbolisches Zeichen dafür, dass der Staat die geltend gemachten Bedrohungsgefühle einer beunruhigten Bevölkerung aufnimmt und bestrebt ist, deren «Sicherheit» zu schützen.

81 Ebenfalls kritisch in diesem Punkt Hug/Scheidegger (Fn. 71), Art. 221 N 39b; ähnlich auch Manfrin (Fn. 36), S. 148.

82 Ähnlich auch Coninx/Mona (Fn. 16), S. 12.

\section{Die präventive verdeckte Polizeitätigkeit}

\section{a) Allgemeine grundrechtliche Problematik}

38 Heute ist generell anerkannt, dass die Tätigkeit verdeckter Ermittler verschiedene grundrechtliche Positionen und elementare prozessuale Rechte der Beschuldigten schwerwiegend beeinträchtigt. ${ }^{83}$ Es handelt sich vor allem um Eingriffe in die persönliche Freiheit, konkret in die Privat- und Geheimsphäre. Daneben wird auch das Recht der Aussageverweigerung (Selbstbelastungsfreiheit, sog. Nemo-tenetur-Prinzip) verletzt, indem die Zielpersonen einer Art von verdecktem Verhör ausgesetzt sind. ${ }^{84}$ Problematisch ist dabei nicht primär die fehlende Offenheit der Ermittlungen, sondern das täuschende Verhalten der Beamten gegenüber den Zielpersonen, d.h. den späteren Beschuldigten. Aus den erwähnten Gründen erfordern sowohl die verdeckte Ermittlung wie auch die verdeckte Fahndung eine gesetzliche Grundlage. ${ }^{85}$ Hierfür stehen derzeit die Art. $285 a$ ff. und Art. $298 a$ ff. StPO zur Verfügung.

39 Mit der Schaffung einer legalen Basis ist die materielle Problematik der verdeckten Ermittlung und Fahndung allerdings nicht beseitigt. Allein schon der zum

83 So z.B. Pieth (Fn. 12), 181 und Tanja Knodel, in: Basler Kommentar, Schweizerische Strafprozessordnung, hrsg. von M.A. Niggli/M. Heer/H. Wiprächtiger, 2. Aufl., Basel 2014, Art. 285a N 1, mit Hinweisen; ferner Zerbes (Fn. 20), S. 466.

84 Siehe insb. Pieth (Fn. 12), S. 177 und Sabine Gless, Strafverfolgung im Internet, ZStrR 2012, S. 16; weiterführend dazu Frank Meyer, Das zulässige Mass beim Zwang - Grenzen zulässigen Verhaltens bei verdeckter Fahndung und Ermittlung, ZStrR 2016, S. $453 \mathrm{ff}$.

85 Vgl. BGE 134 IV 266 E. 3.6.4 S. 276 f. 
Ausdruck gelangende unheimliche Weg in die Heimlichkeit bietet hinreichenden Anlass, die rechtsstaatliche Skepsis nicht zu verdrängen. Weil sich die Betroffenen gegen die verdeckten Massnahmen (naturgemäss) nicht wehren können, ist das Missbrauchspotenzial hoch einzuschätzen; 86 das trifft selbst dort zu, wo das Gesetz eine richterliche Genehmigung verlangt. ${ }^{87}$ Täuschungsmanöver des Staates gegenüber (tatverdächtigen) Personen bleiben infolgedessen allemal bedenklich, und zwar unabhängig von dem damit verbundenen Aufwand. Täuschendes Verhalten seitens der Strafverfolgungsbehörden widerspricht grundsätzlich den Erfordernissen eines fairen Verfahrens gemäss Art. 6 Ziff. 1 EMRK und wird demzufolge allgemein im Gesetz (Art. 140 Abs. 1 StPO) ausdrücklich als verbotene Methode aufgezählt. Dabei ist namentlich zu beachten, dass Strafverfahren gegen Personen gerichtet sind, die aufgrund der Unschuldsvermutung von Rechts wegen als unschuldig gelten (was in der gegenwärtigen rechtspolitischen Debatte freilich häufig verkannt wird). 88

Insbesondere bei den niederschwelligen gesetzlichen Anforderungen an die verdeckte Fahndung ist die Eingriffsintensität regelmässig verharmlost worden. ${ }^{89}$ So bedarf es für diese Art der Ermittlung keiner richterlichen Genehmigung, und sie soll gerade auch - und zwar ungeach-

86 Pieth (Fn. 12), S. 161.

87 So deutlich Oberholzer (Fn. 2), S. $229 \mathrm{ff}$.

88 Peter Albrecht, «Veruntreuungsfalle» - eine verdeckte Ermittlung?, AJP 2012, S. 131, mit Literaturhinweisen.

89 Siehe Bericht der Kommission für Rechtsfragen des Nationalrates vom 3. Februar 2012 zur parlamentarischen Initiative, Präzisierung des Anwendungsbereichs der Bestimmungen über die verdeckte Ermittlung (BBl 2012 5591), S. 5591 ff. und 5603 ff. sowie Lentjes Meili (Fn. 16), S. 450. tet des Grundsatzes der Verhältnismässigkeit (Art. $298 b$ Abs. 1 lit. b StPO) beim Verdacht von Bagatelldelikten zum Zuge kommen (praktisch relevant vor allem bei Scheinkäufen im Drogenkleinhandel90). Ausserdem muss man sich fragen, inwiefern für eine Strafuntersuchung solche Methoden wirklich unentbehrlich sind. 91

\section{b) Präventiver Blickwinkel}

41 In der Praxis weisen Polizeikreise immer wieder mit Nachdruck auf die Unentbehrlichkeit präventiver verdeckter Aktionen hin92. Damit stellt sich unweigerlich die Frage, inwiefern eine (ausschliesslich) auf die Zukunft hin gepolte Sichtweise im geltenden Prozessrecht verankert ist.

42 Sowohl die strafprozessuale verdeckte Ermittlung wie auch die verdeckte Fahndung setzen einen (hinreichenden) Tatverdacht voraus (Art. 286 Abs. 1 lit. a und Art. $298 b$ Abs. 1 lit. a, jeweils i.V.m. Art. 197 Abs. 1 lit. b StPO) 93 und sind insoweit repressiv ausgerichtet. Hingegen enthält die Prozessordnung «keine Bestimmungen zur präventiven Vorermittlung im Sinne eines polizeilichen Tätigwerdens zur Verhinderung oder Erkennung zukünftiger möglicher Delikte (...)».94 Trotzdem finden bei den beiden erwähnten Zwangsmassnahmen nicht selten auch präventive Anliegen eine wesentliche Berücksichtigung.

$90 \overline{\text { Vgl. Thomas Hansjakob, Die neuen Bestimmun- }}$ gen zu verdeckter Fahndung und Ermittlung, forumpoenale 2013, S. $221 \mathrm{f}$.

91 Ebenfalls skeptisch Pieth (Fn. 12), S. 181.

92 So etwa dezidiert Lentjes Meili (Fn. 16), S. 439.

93 Zur verdeckten Ermittlung Botschaft (BBl 2006, Fn. 46), S. 1256 und zur verdeckten Fahndung Bericht (BBl 2012, Fn. 89), S. 5596.

94 BGE 140 I 353 E. 5.5.1 S. 362; ebenso BGE 143 IV 27 E. 2.5 S. 32. 
43 Das wird einmal dort augenfällig, wo die materiell-rechtlichen Normen ihrerseits zukunftsorientiert ausgestaltet sind. Wenn nämlich beispielsweise die Strafbarkeit bereits in einem frühen Stadium der Tatvorbereitung beginnt, sinkt entsprechend auch die Schwelle zum Tatverdacht, den die prozessualen Zwangsmassnahmen erfordern. Hierfür sei an die praktisch sehr bedeutsame Bestimmung der strafbaren Vorbereitungshandlung gemäss Art. 19 Abs. 1 lit. g BetmG erinnert. Auch wenn es in solchen Fällen den Behörden häufig um die Verhinderung des vorbereiteten Betäubungsmittelverkaufs oder -imports (und nicht um die Bestrafung des Täters) geht, kann formal betrachtet - ein hinreichender Tatverdacht für eine verdeckte Ermittlung vorliegen.

44 Andererseits muss man sich zusätzlich vergegenwärtigen, dass nach vorherrschender Ansicht für die verdeckte Fahndung ein bloss «vager Verdacht» (begangener) strafbarer Handlungen genügen soll.95 Diese Haltung ermöglicht einen frühzeitigen Einsatz der Massnahme und fördert somit präventiv motivierte Fahndungshandlungen in einem strafprozessualen «Mantel». 96

Unbestritten ist heute, dass präventive Vorermittlungen ohne bestehenden Tatverdacht sich nicht auf das Bundesrecht abstützen können, sondern in den kantonalen Polizeigesetzen zu regeln sind.97 Insoweit hat die verfassungsrechtliche Kompetenzvorgabe hier (anders als bei

$95 \overline{\text { So Bericht (BBl 2012, Fn. 89), S. 5596; ebenso }}$ BGE 140 I 353 E. 5.4 S. 362.

96 Vgl. Hassemer (Fn. 9), 118; ferner daran anschliessend Zerbes (Fn. 9), 248.

97 Siehe BGE 140 I 353 E. 5.5.2 S. 363 sowie Knodel (Fn. 83), Art. 286 N 17a und Art. $298 b$ N 6. der Untersuchungshaft ${ }^{8}$ ) Beachtung gefunden. Allerdings bezweckt die präventive verdeckte Ermittlung oder Fahndung gemäss den betreffenden Erlassen - entgegen den sonst üblichen Grundsätzen der Polizeitätigkeit - nicht die Abwehr unmittelbar drohender Gefahren. Vielmehr geht es dabei ganz allgemein um die «Verhinderung und Erkennung von Straftaten».99. Aus den völlig offen gehaltenen Gesetzestexten ergibt sich nicht, welche Art von Straftaten behördlich verhindert oder erkannt werden soll. Der Polizei wird also eine ausgedehnte und rechtlich entgrenzte Befugnis für verdeckte Aktionen eingeräumt. Damit verbindet sich das erhebliche Risiko unerlaubter Verdachtsausforschung, namentlich mit Blick auf die Beweissammlung im Internet (konkret in Chaträumen). ${ }^{100}$ Ausserdem fragt sich hier, wie es den zuständigen Behörden im Einzelfall überhaupt möglich ist, konkret und zuverlässig künftige Delikte vorauszusehen (die dann zu verhindern sind).

\section{Schlussfolgerungen}

\section{Zusammenfassung}

46 Im Mittelpunkt meiner mehrfach geäusserten Bedenken gegen die präventive Ausrichtung prozessualer Zwangsmassnahmen steht die zunehmende Ausdehnung der Funktionen des Strafprozesses.

98 Vorne Ziff. III/2/b.

99 So im Kanton Zürich § 32e Abs. 1 des Polizeigesetzes (PolG, 550.1); ähnlich auch z.B. im Kanton SG $\S \S 52^{\text {quater }}$ und $52^{\text {septies }}$ des Polizeigesetzes (PG, 451.1) oder im Kanton Basel-Stadt §§ 33a Abs. 1 und 33b Abs. 1 des Polizeigesetzes (PolG, 510.100).

100 Dazu Pascal Ronc/Sandra van der Stroom, Das Ende der verdeckten Ermittlung im Internet Besprechung des Urteils BGE 143 IV 27, forumpoenale 2017, S. 346. 
Die Untersuchungshaft sowie die verdeckte Ermittlung und Fahndung dienten bisher in erster Linie der Aufklärung von (vermutlich) begangenen Straftaten. Demzufolge bildete der konkrete Tatverdacht den rechtlich massgebenden (vergangenheitsorientierten) Anknüpfungspunkt.

Neuerdings jedoch verlassen die genannten Massnahmen teilweise die herkömmliche repressive Grundlage. Sie übernehmen immer öfters auch Aufgaben für die Zukunft, die generell der Polizei zustehen, nämlich die Verhinderung vermuteter Delikte (als Gefahrenabwehr). Besonders augenfällig wird die Schwerpunktverlagerung beim Haftgrund der Ausführungsgefahr (Art. 221 Abs. 2 StPO), wo der Gesetzgeber auf das Erfordernis eines Tatverdachts bewusst verzichtet hat. Abgesehen davon verlangen die strafprozessualen Interventionen auf der Basis präventiver Haftgründe keine konkrete und unmittelbare Gefahrenlage. Insofern senken sie - abweichend von der polizeirechtlichen Maxime - die Eingriffsschwelle. ${ }^{101}$ Erschwerend kommt noch hinzu, dass die strafprozessuale Präventivhaft $\mathrm{zu}$ Freiheitsentzug in einem Ausmass führt, das im Polizeirecht bislang unvorstellbar ist. ${ }^{102}$ Zum andern entfernt sich die kantonale Polizeigesetzgebung ihrerseits ebenfalls von den eigenen Prinzipien, wenn sie Bedürfnisse der Strafverfolgung übernimmt und folglich für ihre Massnahmen der präventiven verdeckten Ermittlung oder Fahndung keine akute Bedrohung von Rechtsgütern voraussetzt. ${ }^{103}$ Diese legislativen Entwicklungen konvergieren in Richtung ei-

${ }_{101}$ Siehe vorne Ziff. III/2/c/bb.

${ }_{102}$ Siehe vorne Ziff. III/2/c/bb.

${ }_{103}$ Siehe vorne Ziff. III/3/b. ner weit gefassten und konturenlosen Gefahrenabwehr mit entsprechenden Folgen für die Freiheitsrechte der betroffenen Personen.

\section{Mut zur Lücke!}

48 Die eben geschilderten Funktionserweiterungen sowohl des Strafverfahrens als auch der Polizeitätigkeit vermitteln deutliche Anzeichen eines beabsichtigten flächendeckenden Rechtsgüterschutzes. Ungeachtet der in der Verfassung festgeschriebenen Kompetenzordnung erhalten die Behörden durch die Gesetzgebung immer mehr Befugnisse, mittels Zwangsmassnahmen gegen Personen seien es Tatverdächtige oder Störer vorzugehen. Auf diese Weise reduziert sich indessen zwischen Verdachtsabklärung und Gefahrenabwehr zusehends der Freiheitsspielraum der Individuen. Die bislang als unverzichtbar erachteten Bollwerke gegen behördliche Eingriffe erleiden massive Erschütterungen. So werden einerseits (für das Strafverfahren) der konkrete Verdacht eines Deliktes und andererseits (für die Polizeitätigkeit) die Abwehr einer akuten Gefahr als Eingriffsvoraussetzungen abgeschliffen oder gar ganz beseitigt. Durch die hier zu beobachtenden Verschiebungen in den Fundamenten der Sicherheitsarchitektur werden die staatsfreien Zonen immer weiter zubetoniert ${ }^{104}$.

49 Wem die Freiheitsidee im Strafrecht ein wirklich ernsthaftes Anliegen ist, der muss Widerstand leisten gegenüber dem gegenwärtigen Trend zu einer möglichst lückenlosen Palette von Zwangsmassnahmen. Diese Instrumente ziehen der-

$104 \overline{\text { Sehr instruktiv dazu Denninger (Fn. 8), S. } 90 \text { und }}$ Zerbes (Fn. 9) 246. 
zeit ungeordnet kreuz und quer durch das Strafprozess- und das Polizeirecht ohne hinreichende Rücksichtnahme auf die verfassungsrechtlichen Vorgaben und ohne eine rechtsdogmatische Konsistenz. Dabei bleibt das elementare Prinzip der Verhältnismässigkeit (Art. 197 Abs. 1 lit. c und d StPO) nur allzu oft auf der Strecke. Gerade bei den unter dem Dach der Prävention angesiedelten Interventionen kommt noch hinzu, dass sie jeweils mit schwer lösbaren Prognoseschwierigkeiten belastet sind, was leicht willkürliche Entscheide zum Nachteil der betroffenen Personen bewirkt. Aus liberaler Sicht sollten deshalb die Gesetzgebung und die Rechtsprechung sich vor einem Sturz in den Strudel einer unreflektierten Sicherheitspolitik hüten und sich gegen grenzenlose Freiheitseingriffe einsetzen. Gefordert sind deutlich mehr Resistenz, mehr Mut zur Lücke in der strafprozessualen Dimension des Rechtsgüterschutzes. ${ }^{105}$

$105 \overline{\text { Parallel dazu steht auf der materiell-rechtlichen }}$ Ebene die Forderung nach einem fragmentarischen Strafrecht. 